\title{
Unilateral Substantia Nigra Lesions and Schedule-Induced Polydipsia
}

\author{
GUY MITTLEMAN AND ELLIOT S. VALENSTEIN ${ }^{1}$ \\ Department of Psychology and Neuroscience Laboratory Building, The University of Michigan \\ 1103 E. Huron St., Ann Arbor, MI 48104-1687
}

Received 25 February 1985

\begin{abstract}
MITTLEMAN, G. AND E. S. VALENSTEIN. Unilateral substantia nigra lesions and schedule-induced polydipsia. PHYSIOL BEHAV 36(3) 437-440, 1986.- The present experiment investigated the effects of unilateral 6-hydroxydopamine lesions of the substantia nigra (SN) on schedule-induced polydipsia (SIP). Lesions were made in either the "dominant" or "non-dominant" hemisphere as defined by an amphetamine rotation test. It was found that unilateral lesions of either the "dominant" or "non-dominant" SN significantly reduced SIP and also significantly impaired somatosensory responsiveness as indicated by the "tactile extinction test." Somatosensory neglect was significantly greater following a lesion in the "dominant" hemisphere than "non-dominant" hemisphere.
\end{abstract}

Substantia nigra Schedule-induced polydipsia Amphetamine Rotation Somatosensory neglect

WHEN food deprived animals are given small amounts of food reward, intermittently, they consume excessive amounts of water and under some conditions may drink as much as half their body weight in 3 hours [5]. Excessive drinking under these conditions cannot be adequately explained in terms of physiological homeostasis or as a result of the adventitious reinforcement of drinking by food reward $[4,5,30]$. Schedule-induced polydipsia (SIP) as well as other schedule-induced behaviors, including attack, wheel running, pica eating, and air-licking occur when a strongly motivated appetitive behavior is interrupted or prevented and may be a laboratory analogue to the displacement behaviors described by ethologists [5]. It has been suggested that schedule-induced behaviors occur because the motivational excitement or activation that accompanies the delivery of a food reward, outlasts its consumption, and potentiates alternative activities evoked by available environmental stimuli [13].

The activational effect of a food reward in this situation has been compared to the behavioral activation produced by electrical stimulation of the lateral hypothalamus (ESLH) [3, 27, 28, 29]. Indeed, the behaviors elicited by ESLH are strikingly similar to behavior that is schedule-induced and it has recently been demonstrated that animals which eat and drink during ESLH display the greatest amount of SIP [15]. That SIP- and ESLH-elicited ingestive behavior may be related to common mechanisms is supported by evidence that behaviors evoked under both conditions depend on dopamine pathways $[2,17,18,19,20,21,26,27]$. Most recently, it was demonstrated that unilateral 6-hydroxydopamine (6-OHDA) lesions of forebrain dopamine systems significantly attenuated ESLH-elicited eating and drinking [16]. The purpose of the current experiment was to determine the effects of unilateral substantia nigra lesions of either the "domi- nant" or "non-dominant" hemisphere on SIP and on somatosensory responsiveness.

\section{METHOD}

Subjects

The subjects were 32 mature (356-468 g), male, Long Evans hooded rats (Simonsen Co., Gilroy, CA) housed in wire hanging cages with free access to food and water. The vivarium was temperature controlled and the animals were maintained on a 12-12 hr dark-light cycle.

\section{Pre-operative Testing}

Rats were tested for amphetamine-induced rotational behavior to determine the striatum with greater dopamine activity [8]. It has previously been shown that the striatum contralateral to an animal's preferred direction of rotation has higher dopamine levels and greater metabolic activity $[7,9]$. Rotational behavior was measured by automated spherical rotometers similar to those described by Greenstein and Glick [10].

Each rat was placed in the rotometer for a 15 minute habituation period and then injected with $1.2 \mathrm{mg} / \mathrm{kg}$ $\mathrm{d}$-amphetamine sulfate (AMPH) dissolved in $0.9 \%$ saline. AMPH-induced rotational behavior was recorded for 60 minutes. Based on the majority of full rotations (a rotation consists of 4, 90 degree turns in the same direction), the hemisphere contralateral to the preferred direction of rotation was designated the "dominant," and the other the "non-dominant" hemisphere. Only rats that made at least $70 \%$ of their total rotations in one direction (i.e., $70 \%$ dominant) and a minimum of 10 full rotations in that direction were used as subjects.

\footnotetext{
'Requests for reprints should be addressed to Professor Elliot S. Valenstein.
} 


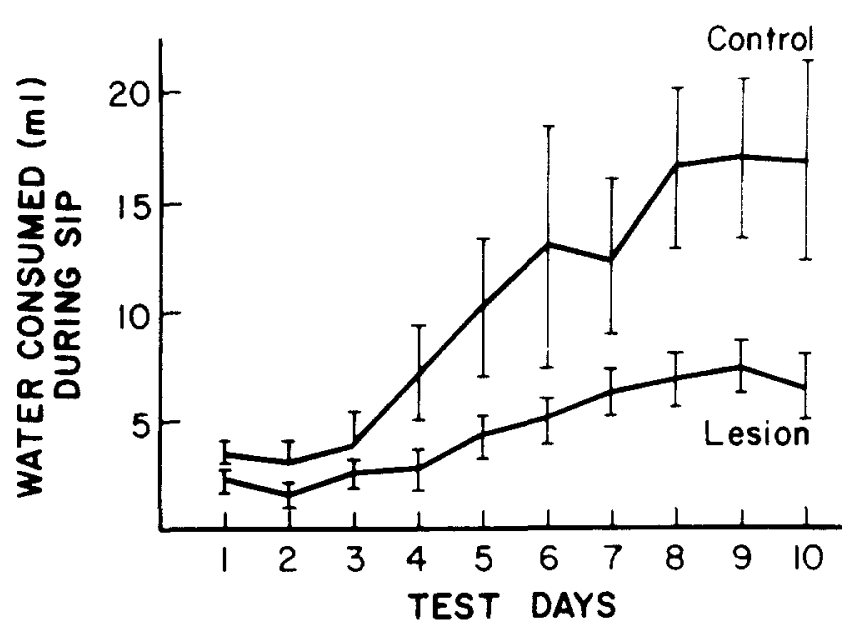

FIG. 1. Mean ( \pm S.E.M.) amount of water consumed in each schedule-induced polydipsia test in lesioned and control rats. The data from the animals with dominant and non-dominant hemisphere lesions did not differ and were, therefore, combined.

One week after the $\mathrm{AMPH}$-induced rotation test, rats were tested for asymmetry in sensorimotor responsiveness using the "tactile extinction test" described by Schallert 't al. [24]. A small piece of sticky tape (Pres-a-Ply Label, Dennison) was simultaneously placed on the radial aspect of the animal's forepaws and the latency to remove each label was recorded. This test is analogous to the "simultaneous stimulation test" used clinically with patients recovering from brain damage $[12,25]$.

\section{Surgery}

The day after the "tactile extinction test" was administered, each rat was anaesthetized with Equithesin (JensenSalsbery, Kansas City, MO) and received a $25 \mathrm{mg} / \mathrm{kg}$ (IP) injection of desipramine [1]. Thirty minutes later a 30 gauge stainless steel infusion cannula was placed unilaterally into the zona compacta of the substantia nigra (coordinates: 5.0 $\mathrm{mm}$ posterior to bregma; $2.0 \mathrm{~mm}$ lateral to the midsagittal suture; and $8.0 \mathrm{~mm}$ below the surface of the skull which was level between bregma and lambda). Destruction of dopamine neurons was accomplished by infusion of 6-hydroxydopamine $(6-O H D A ; 8 \mu \mathrm{g} / 2 \mu \mathrm{l})$ dissolved in $0.9 \%$ saline with $0.1 \%$ ascorbic acid, at a rate of $1 \mu \mathrm{l} / \mathrm{min}$. Rats received a lesion in the "dominant" $(\mathrm{N}=14)$ or "non-dominant" $(\mathrm{N}=11)$ hemisphere. Animals in the control group $(\mathrm{N}=7)$ were treated identically except an empty cannula was placed unilaterally into the substantia nigra.

\section{Post-operative Testing}

Ten days following surgery when all animals had surpassed their baseline weight, they were retested for sensorimotor responsiveness using the "tactile extinction test" as described above. Rats were then food deprived until they reached $85 \%$ of their baseline weight and given 10 daily tests for schedule-induced drinking in a $30 \times 30 \times 46 \mathrm{~cm}$ high Plexiglas chamber. A food cup connected to a pellet dispenser was located in the center of one wall. A Plexiglas partition that protruded $7 \mathrm{~cm}$ into the center of the test chamber was located on each side of the food cup. A water filled Richter
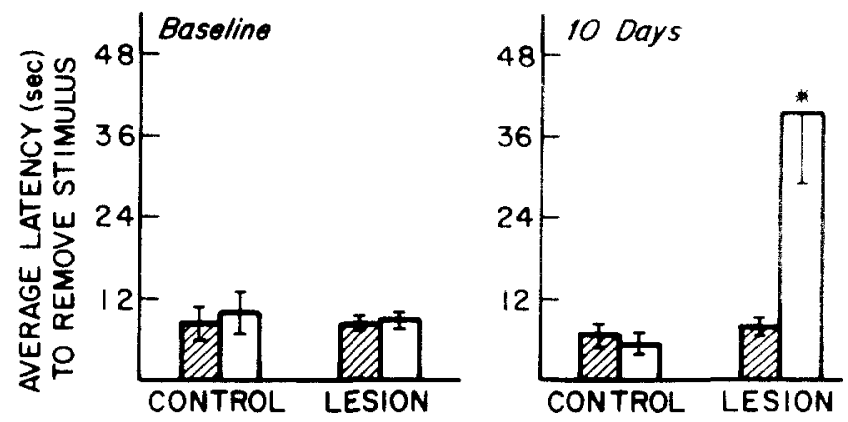

FIG. 2. Results of the tactile extinction test administered before (baseline) and 10 days after a unilateral substantia nigra lesion. I aitency to remove the adhesive label from the radial aspect of the forelimb contralateral (open bar) and ipsilateral (striped bar) to a unilateral 6-OHDA lesion of the substantia nigra. *Significant at $p<0.01$ (paired $t$-test).

tube with a photocell drinkometer was located $5 \mathrm{~cm}$ on each side of the food cup and the amount of water consumed was recorded at the end of each test session. The water consumed was recorded separately from the Richter tubes located on either side of the food cup. During the first four daily tests, a drinkometer was used to record the rate of licking water. A food pellet (P. J. Noyes, $75 \mathrm{mg}$ ) was automatically dispensed every 60 seconds of the 30 minute test. during which time behavior was continuously monitored on closed-circuit television.

\section{Neurochemical Methods}

Between 7 and 10 days after the completion of all behavioral testing, rats in the experimental and control groups were decapitated. The brain of each rat was rapidly removed [11] and placed in ice cold saline and prepared for assay of dopamine (DA) using a method modified from Felice $\iota t a l$. [6]. Tissue concentrations of DA were measured by high performance liquid chromotography with electrochemical detection (HPLC-EC).

\section{RESULTS}

The results clearly demonstrate that unilateral 6-OHDA lesions of the substantia nigra significantly attenuated the amount of schedule-induced drinking displayed (Fig. 1). During SIP testing, lesioned rats drank significantly less water than animals in the control group (ANOVA: $F(1,26)=8.59$, $p=0.0069$ ). The difference in development of SIP across the 10 test days is obvious in Fig. 1. Schedule-induced drinking increased at a significantly slower rate in lesioned rats (ANOVA: $F(2,234)=6.01, p<0.001$ ). Cumulative-pen recordings made on test days 5 and 10 indicated that for rats in both groups, drinking occurred predominantly in the time immediately after each pellet delivery, the normal temporal locus of most schedule-induced drinking [5]. There were no significant differences in the total amount of water consumed by rats lesioned in the "dominant" $(x=47.4 \pm 11.7 \mathrm{ml})$ or "non-dominant" $(x=36.4 \pm 7.8 \mathrm{ml})$ hemisphere and their results are combined in Fig. 1. Moreover, there was no difference in the amount of water consumed from either Richter 
tube so these data were also combined. All rats in the lesioned as well as the control groups ate all of the pellets during the SIP tests immediately after they were dispensed. During the 7-10 days following completion of the SIP tests, when food and water were provided ad lib, animals in the lesioned and the control groups gained body weight at the same rate.

The results of the first "tactile extinction test," administered before the lesion, indicated that there was no difference in the latency to remove tape from either forepaw between the groups designated "control" and "lesion" (Fig. 2 ). In comparison, at 10 days after surgery there was a significant increase in the latency to remove tape from the contralateral forepaw of lesioned rats, $\mathrm{t}(22)=3.25, p<0.01$. As expected, tape removal time in control animals did not increase following surgery, and actually decreased slightly, presumably due to learning.

In agreement with a previous report $[16,22]$, lesions of the "dominant" hemisphere were significantly more effective in producing changes in the latency scores in the "tactile ex. tinction test," $t(22)=2.19, p<0.05$. Rats lesioned in the "dominant" hemisphere had a latency of $49.7 \mathrm{sec}( \pm 8.1)$ to remove tape placed on the contralateral forepaw while the latency of "non-dominant" lesioned rats was $24.5 \mathrm{sec}$ $( \pm 5.1)$. Neither "dominant" nor "non-dominant" lesions altered the latency to remove the tape on the ipsilateral paw.

Following unilateral 6-OHDA lesions of the substantia nigra, the biochemical analysis indicated that dopamine was depleted by $89 \%$ in the striatum and $61 \%$ in the nucleus accumbens, compared to the non-lesioned side. There were no significant differences in DA depletion in rats lesioned in the "dominant" or "non-dominant" hemisphere.

\section{DISCUSSION}

The results of this experiment demonstrate that unilateral depletion of DA in the striatum and nucleus accumbens significantly reduced schedule-induced drinking although it apparently had no effect on ad lib eating or drinking. This finding extends previous reports concerning the effects of bilateral dopaminergic lesions in attenuating SIP $[21,27]$.
It seems unlikely that the reduction in schedule-induced drinking following unilateral lesions of the substantia nigra can be explained by any motor impairment. No motor impairment was observed and Robbins and Koob [21] reported that following bilateral lesions of the mesolimbic DA pathway, there was only a minimal reduction in lick-efficiency (licks/ml water consumed) observed and this was not sufficient to account for the reduction in SIP. In the current experiment, lick records obtained during the first 4 test days indicated that lesion and control animals did not differ significantly in the number of licks/ml water. It seems unlikely, therefore, that the attenuation in SIP following the unilateral lesions was due to an induced motor disability.

There was no evidence that these unilateral lesions attenuated SIP by reducing the motivating aspects of food deprivation. As noted, all rats ate every food pellet almost immediately upon its delivery and lesioned animals actually spent more time in the vicinity of the food cup $(x=5.54$ $\mathrm{min} / \mathrm{SIP}$ test $)$ than non-lesioned rats $(4.57 \mathrm{~min})$. In addition, all animals immediately ate the supplementary food placed in their home cages after each SIP test and they also drank the water. Thus, we found that the dramatic reduction in schedule-induced drinking was not paralleled by an effect on ad lib ingestive behavior.

We believe the present results are best explained by the effect of the lesion in reducing either the level or duration of the activation that normally occurs following intermittent delivery of food to hungry animals. The drinking and also eating that is often evoked when animals are activated has been called "non-regulatory ingestive behavior" by the present authors [16]. A considerable amount of evidence is accumulating that this activation and ingestive behavior are regulated by forebrain dopamine systems.

\section{ACKNOWLEDGEMENTS}

The authors are pleased to acknowledge the cooperation of Dr. T. E. Robinson in permitting us to use his automated rotometers and Dr. J. B. Becker for the use of the HPLC system.

\section{REFERENCES}

1. Breese, G. R. and T. D. Traylor. Depletion of brain noradrenaline and dopamine by 6-hydroxydopamine. Br J Pharmacol 42: 88-89, 1971 .

2. Brett, L. P. and S. Levine. Schedule-induced polydipsia suppresses pituitary-adrenal activity in rats. I Comp Physiol Psychol 5: 946-956, 1979.

3. Cantor, M. B. and J. F. Wilson. Polydipsia induced by a schedule of brain stimulation reinforcement. Learn Motivation 9: $428-445,1978$

4. Falk, J. L. Conditions producing psychogenic polydipsia in animals. Ann NY Acad Sci 157: 569-593, 1969.

5. Falk, J. L. The nature and determinants of adjunctive behavior. Physiol Behav 6: 577-588, 1971.

6. Felice, L. J., J. D. Felice and P. T. Kissinger. Determination of catecholamines in rat brain parts by reverse-phase ion-pair liquid chromatography. J Neurochem 31: 1461-1465, 1978.

7. Glick, S. D., T. P. Jerussi, D. H. Waters and J. P. Green. Amphetamine-induced changes in striatal dopamine and acetylcholine levels and relation to rotation (circling behavior) in rats. Biochem Pharmacol 23: 3223-3225, 1974.

8. Glick, S. D., T. P. Jerussi and B. Zimmerberg. Behavioral and neuropharmacological correlates of nigrostriatal asymmetry in rats. In: Lateralization in the Nervous System, edited by $\mathrm{S}$. Harnad et al. New York: Academic Press, 1977, pp. 213-249.
9. Glick, S. D., R. C. Meibach, R. D. Cox and S. Maayani. Multiple and interrelated functional asymmetries in rat brain. Life $S \mathrm{C} i$ 25: 395-400, 1979.

10. Greenstein, S. and S. D. Glick. Improved automated apparatus for recording rotation (circling behavior) in rats and mice. Pharmacol Biochem Behav 3: 507-510, 1975.

11. Heffner, T. G., J. A. Hartman and L. S. Seiden. A rapid method for the regional dissection of the rat brain. Pharmacol Biochem Behav 13: 453-456, 1980.

12. Heilman, K. M. Neglect and related disorders. In: Clinical Neuropsychology, edited by K. M. Heilman and E. S. Valenstein. New York: Oxford Press, 1979, pp. 268-307.

13. Killeen, P. R., S. J. Hanson and S. R. Osbourne. Arousal: Its genesis and manifestation as response rate. Psychol Rev 85: 571-581, 1978.

14. Koob, G. F., S. J. Riley, C. Smith and T. Robbins. Effects of 6-hydroxydopamine lesions of the nucleus accumbens septi and olfactory tubercle on feeding, locomotor activity and amphetamine anorexia in the rat. J Comp Physiol Psychol 5: 917$927,1978$.

15. Mittleman, G. and E. S. Valenstein. Ingestive behavior evoked by hypothalamic stimulation and schedule-induced polydipsia are related. Science 224: 415-417, 1984. 
16. Mittleman. G., P. J. Fray and E. S. Valenstein. Asymmetry in the effects of unilateral 6-OHDA lesions on eating and drinking evoked by hypothalamic stimulation. Behat Brain Re's 15: 263267, 1985

17. Phillips, A. G. and H. C. Fibiger. Deficits in stimulation induced feeding after intraventricular administration of 6-hydroxydopamine in rats. Behar Biol 9: 749-754, 1973.

18. Phillips, A. G. and H. C. Fibiger. Long-term deficits in stimulation-induced behaviors and self-stimulation after 6-hydroxydopamine administration in rats. Behar Biol 16: $127-$ $143,1976$.

19. Phillips, A. G. and H. C. Fibiger. The multivariate nature of motivated behavior elicited by electrical stimulation of the lateral hypothalamus. In: Current Studies of Hypothalamic Functirm, vol 2, edited by W. L. Veale and K. Ledaris. Basel: Karger, 1978, pp. 195-205.

20. Phillips, A. G. and R. S. Nikaido. Disruption of brain stimulation-induced feeding by dopamine receptor blockade. Nature 258: 750-751, 1975.

21. Robbins. T. W. and G. F. Koob. Selective disruption of displacement behaviour by lesions of the mesolimbic dopamine system. Natur' 285: 409-412, 1980.

22. Robinson, T. E., J. B. Becker, D. M. Camp and A. Mansour. Variation in the pattern of behavioral and brain asymmetries due to sex differences. In: Corrbral Lateralization in SubHuman Specie's, edited by S. D. Glick. New York: Academic Press. 1985. pp. 185-231.

23. Rowland, N., D. M. Marques and A. E. Fisher. Comparison of the effects of brain dopamine-depleting lesions upon oral behaviors elicited by tail pinch and electrical brain stimulation. Phrsiol Behar 24: 273-281, 1980.
24. Schallert, T., M. Upchurch, N. Lobaugh, S. B. Farrar, W. W Spirduso, P. Gilliam, D. Vaughn and R. E. Wilcox. Tatctile ex tinction: Distinguishing between sensorimotor and molor asymmetries in rats with unilateral nigrostriatal damage. $P / h u$ macol Bioche'm Be'ha1' 16: 455-462, 1982.

25. Schwartz. A. S., P. L. Marchok and R. E. Flynn. A sensitive test for tactile extinction: resuls in patients with parietal and

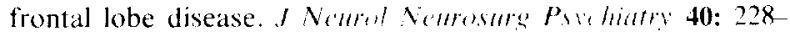
232, 1977 .

26. Valenstein. E. S. Stereotypy and sensory-motor changes evoked by hypothalamic stimulation: Possible relition to schizophrenic behavior patterns. In: Biolegy of Reinfirrement. edited by A. Routtenberg. New York: Academic Press, 1980. pp. 39-52

27. Wallace, M., G. Singer, J. Finlay and S. Gibson. The effect of 6-OHDA lesions of the nucleus accumbens septum on schedule-induced drinking, wheelrunning and corticosterone levels in the rat. Pharmacol Bioch'm Beha' 18: 129-136, 1983.

28. Wayner. M. J. Motor control functions of the lateral hypothalamus and adjunctive behavior. Physiol Behav s: 1319-1325. 1970.

29. Wayner, M. J.. F. C. Barone and C. C. Loullis. The lateral hypothalamus and adjunctive behavior. In: Handhose of the Hypothalamus, iol 3-part B. Beharioral Studis of the Hyoethalumus, edited by P. J. Morgane and J. Panksepp. New York: Marcel Dekker. Inc., 1981, pp. 107-145.

30. Wetherington, C. L. Is adjunctive behavior a third class of behavior?" Nemoser Behav Re' 6: 329-350, 1982. 